\title{
Aleitamento materno e uso de medicamentos durante a lactação
}

\author{
Breastfeeding and use of medications during lactation
}

Luiz Antonio Del Ciampo', Ivan Savioli Ferraz¹, Julio Cesar Daneluzzi', Rubens Garcia Ricco², Carlos Eduardo Martinelli Junior ${ }^{1}$

\section{RESUMO}

Objetivo: Conhecer aspectos da utilização de medicamentos durante o período de aleitamento materno entre mães de crianças com idades iguais ou menores que seis meses.

Métodos: Estudo transversal descritivo, com entrevistas a todas as mães de crianças com idades iguais ou menores que seis meses, atendidas em serviço de atenção básica à saúde durante um período de seis meses, e que, por questionário estruturado, informaram sobre o consumo de medicamentos durante a lactação.

Resultados: Foram entrevistadas 502 mães, das quais 341 $(68 \%)$ referiram ter utilizado um ou mais medicamentos. Destas, 316 (93\%) seguiram prescrição de receitas médicas e $25(7 \%)$ o fizeram por conta própria. Os medicamentos mais utilizados foram sais de ferro e vitaminas (59\%), analgésicos/antitérmicos/antiinflamatórios (15\%), hormônios $(12 \%)$, antibióticos $(7 \%)$, antieméticos $(2 \%)$. Apenas três (1\%) pacientes interromperam o aleitamento materno devido à necessidade da utilização de carbonato de lítio.

Conclusões: Considerando-se que grande parte das lactantes utiliza algum medicamento durante o período de lactação, destaca-se a importância da divulgação de conhecimentos sobre fisiologia humana, bioquímica e das propriedades farmacológicas dos medicamentos, visto que o uso inadequado de fármacos e o desconhecimento de suas relações com o leite humano podem levar a prejuízos, tanto pela passagem do medicamento através do leite para o lactente, como pela interrupção precipitada do aleitamento materno.

Palavras-chave: aleitamento materno; cuidado da criança; atenção primária à saúde.

\section{ABSTRACT}

Objective: Study the profile of medicines used by breastfeeding mothers of infants during the first six months of life.

Methods: This cross-sectional descriptive study enrolled all breastfeeding mothers of infants aged birth to six months, assisted at health care centers during a period of six months. A structured questionnaire regarding medicines used during theses 6-month breastfeeding period was answered by the mothers.

Results: 502 mothers were interviewed and 341 (68\%) of them informed the use of one or more medicines during the first six months of life of their breastfed infants. Of these, 316 (93\%) followed medical prescriptions and $25(7 \%)$ reported over-the-counter use. The most consumed medicines were iron preparations and vitamins (59\%), followed by analgesic/antipyretic/anti-inflammatory drugs (15\%), hormones $(12 \%)$, antibiotics $(7 \%)$ and medication for nausea $(2 \%)$. Only three (1\%) mothers had interrupted breastfeeding due to lithium carbonate treatment.

Conclusions: considering that great part of lactating mothers uses some medicine during breastfeeding, it is important to improve the knowledge of health professionals regarding the relationships between lactation and medicines of common use, in order to reduce damages of inappropriate and inadvertent administration of medications through human milk, as well as to decrease inappropriate and early cessation of breastfeeding.

Key-words: breastfeeding; child care; primary health care.
'Professor doutor do Departamento de Puericultura e Pediatria da Faculdade de Medicina de Ribeirão Preto da Universidade de São Paulo (FMRP-USP), Riberão Preto, SP, Brasil

${ }^{2}$ Professor associado do Departamento de Puericultura e Pediatria da FMRP-USP, Riberão Preto, SP, Brasil

Endereço para correspondência:

Luiz Antonio Del Ciampo

Departamento de Puericultura e Pediatria da FMRP-USP
Avenida Bandeirantes, 3.900

CEP 14049-900 - Ribeirão Preto/SP

E-mail: delciamp@fmrp.usp.br

Financiamento: trabalho desenvolvido com o apoio da Fundação de Amparo ao Estudo, Pesquisa e Assistência do Hospital das Clínicas da FMRP-USP

Recebido em: 5/7/2007

Aprovado em: 19/9/2007 


\section{Introdução}

O leite materno, indiscutivelmente, é o melhor alimento que pode ser oferecido para o lactente, quer seja pelas suas características biológicas e nutricionais ou devido às várias qualidades e benefícios de ordem física e emocional que o aleitamento materno proporciona tanto para a criança quanto para a mãe $e^{(1,2)}$.

A prática do aleitamento materno, principalmente se desenvolvida como maneira exclusiva de alimentar o lactente, deve ser estendida até o sexto mês de vida e, a partir de então, continuada até os dois anos de idade, sendo então complementada com outros alimentos. Considerando-se os múltiplos fatores que a ela estão relacionados para que esta prática seja adequada e naturalmente desenvolvida, é necessário orientação e apoio à lactante e aos familiares - tarefa esta de grande importância e de competência dos serviços de saúde e de toda comunidade, no sentido de incentivar e facilitar o aleitamento materno ${ }^{(3-8)}$.

Torna-se importante, então, identificar e entender todos os fatores que, eventualmente, possam interferir negativamente nesta prática e contribuir para o desmame precoce. Dentre os vários fatores que se relacionam negativamente com o aleitamento materno, encontra-se o uso de medicamentos pela lactante, os quais podem ser incompatíveis ou interferir na alimentação do bebê, reduzindo a produção de leite ou, pior, levando ao desmame. Este último ocorre, pois, em razão do desconhecimento de características farmacológicas, bioquímicas e das diversas etapas do metabolismo dos fármacos no organismo humano, os medicamentos podem ser erroneamente rotulados como contra-indicados durante o aleitamento materno e, devido à indicação precipitada e equivocada de desmame, causar enormes prejuízos à criança e à mãe $e^{(9)}$.

Tabela 1 - Distribuição das características das 502 mães

\begin{tabular}{lrr}
\hline & $\mathbf{N}$ & $\%$ \\
\hline Faixa etária (anos) & 85 & 17 \\
$14-19$ & 287 & 57 \\
$20-29$ & 118 & 24 \\
$30-39$ & 12 & 2 \\
$\geq 40$ & & \\
Ocupação & 356 & 71 \\
$\quad$ Do lar & 146 & 29 \\
$\quad$ Trabalho fora do lar & & \\
Número de filhos & 242 & 48 \\
1 & 129 & 26 \\
2 & 71 & 14 \\
3 & 34 & 7 \\
4 & 26 & 5 \\
5 ou mais & & \\
Escolaridade* & 239 & 48 \\
$\quad$ Primeiro grau & 216 & 43 \\
Segundo grau & 46 & 9 \\
Superior & 502 & 100 \\
Pré-natal & & \\
\hline
\end{tabular}

*1 mãe com escolaridade desconhecida
Assim, o presente trabalho teve como objetivo conhecer alguns aspectos da utilização de medicamentos durante o período de aleitamento materno em mães de crianças com idades iguais ou menores que seis meses.

\section{Métodos}

Trata-se de um estudo do tipo transversal, para o qual foram convidadas a participar todas as mães de crianças com idades variando do nascimento a seis meses completos e que freqüentavam programas de puericultura em unidades básicas de saúde do distrito de saúde oeste da cidade de Ribeirão Preto, São Paulo, ligadas à Faculdade de Medicina de Ribeirão Preto da Universidade de São Paulo (FMRP-USP).

Diariamente, durante seis meses, o propósito do estudo era apresentado às mães que compareciam para atendimento nestas unidades de saúde; eram incluídas aquelas que concordassem em participar, após a obtenção do termo de consentimento livre e esclarecido. Não foram incluídas no estudo crianças levadas para atendimento médico por outro acompanhante que não a própria mãe. Todas as mães convidadas concordaram em participar do estudo.

As mães respondiam a um questionário estruturado, elaborado para o propósito do estudo, com respostas abertas e fechadas, aplicado por um entrevistador treinado (médicos e enfermeiros das equipes de saúde das unidades onde o estudo foi desenvolvido). Neste questionário constava, entre outros dados, perguntas sobre o consumo de medicamentos durante o período de lactação e, quando utilizados, quem indicou os medicamentos (receita médica, farmacêutico, outras pessoas ou por conta própria). Por se tratar de um estudo apenas descritivo, não foi realizado nenhum tratamento estatístico dos dados. O estudo foi aprovado pelo Comitê de Ética em Pesquisa do Hospital das Clínicas da FMRP-USP.

\section{Resultados}

Foram entrevistadas 502 mães, das quais 341 (68\%) referiram ter utilizado um ou mais medicamentos durante o período de aleitamento materno. Destas, 316 (93\%) seguiram a prescrição de receitas médicas e 25 (7\%) o fizeram por conta própria. Nenhuma das entrevistadas seguiu orientação de outras pessoas ou profissionais de saúde que não fossem médicos.

Dentre o grupo constituído por 502 mães, pôde-se verificar que a maior parte delas tinha entre 20 e 40 anos de idade (81\%), eram donas-de-casa $(71 \%)$, com um ou dois filhos $(74 \%)$, escolaridade até o segundo grau $(91 \%)$ e que todas fizeram seguimento pré-natal em unidades básicas de saúde, conforme pode ser observado na Tabela 1. 
Quanto à utilização de medicamentos durante o período de aleitamento materno, encontraram-se 434 respostas afirmativas para as 341 lactantes que o fizeram, visto que, em vários casos, as mães referiram ter utilizado mais de um tipo de fármaco. De acordo com a Tabela 2, vitaminas e sais de ferro (59\%) foram os medicamentos mais utilizados pelas lactantes, justificados por prescrição de rotina dos serviços para suplementação alimentar da mãe no período pós-parto e durante a lactação. Os medicamentos mais consumidos para o tratamento de doenças ou como sintomáticos integraram o grupo dos analgésicos/antitérmicos/antiinflamatórios (15\%), hormônios (12\%), anticoncepcionais (2\%) e antieméticos (2\%). O estudo encontrou apenas três (1\%) pacientes que interromperam o aleitamento materno devido à necessidade da utilização de carbonato de lítio para tratamento psiquiátrico.

\section{Discussão}

Os resultados evidenciam que grande parte das lactantes entrevistadas (41\%) utilizou algum tipo de fármaco durante o período de lactação. Estudos mostram que, em alguns países, a prevalência de lactantes que utilizam medicamentos durante $o$ período de aleitamento materno pode chegar a $90 \%$, considerando-se tanto as prescrições médicas quanto a auto-medicação, sendo, portanto, um fato que deve ser conhecido e considerado no momento das orientações para implantação e manutenção do aleitamento materno entre as puérperas ${ }^{(9-12)}$.

Observa-se que os suplementos com ferro e as vitaminas foram os medicamentos mais utilizados e, em algumas ocasiões, além do efeito suplementar primário, podem também eventualmente apresentar, como efeito placebo, estimulo à amamentação, conforme relatado por algumas mães que referiram o motivo da utilização do medicamento.

Excetuando-se os casos de uso do carbonato de lítio, que contra-indica o aleitamento materno, todos os outros grupos de medicamentos utilizados eram compatíveis com a amamentação. Deve-se destacar que também alguns sintomas, como

\section{Referências bibliográficas}

1. Teruya K, Coutinho SB. Sobrevivência infantil e aleitamento materno. In: Rego JD, editor. Aleitamento materno. $2^{\mathrm{a}}$ ed. São Paulo: Atheneu; 2006.

2. Schack-Nielsen L, Michaelsen KF. Breast feeding and future health. Curr Opin Clin Nutr Metab Care 2006;9:289-96.

3. WHO - Department of Nutrition for Health and Development. Report of the expert consultation on the optimal duration of exclusive breastfeeding. Geneve: WHO; 2002.

4. Zetterström R. Initiation of breastfeeding. Acta Paediatr Suppl 2003;91:9-11.

5. Faleiros FT, Trezza EM, Carandina L. Factors influencing breastfeeding decision and duration. Rev Nutr 2006;19:623-30.

6. Ministério da Saúde. Dez passos para uma alimentação saudável: guia alimentar para crianças menores de 2 anos - Série A - normas e manuais técnicos 107. Brasília: Ministério da Saúde/OPAS; 2002.

7. Lewallen LP, Dick MJ, Flowers J, Powell W, Zickefoose KT, Wall YG et al. Breastfeeding support and early cessation. J Obstet Gynecol Neonatal Nurs. 2006;35:166-72
Tabela 2 - Distribuição dos grupos de medicamentos utilizados

\begin{tabular}{lrc}
\hline Grupo de medicamentos & N & \% \\
\hline Ferro/vitaminas & 257 & 59 \\
Analgésico/antitérmico/antiinflamatório & 66 & 15 \\
Hormônios & 52 & 12 \\
Antibióticos & 30 & 7 \\
Antieméticos & 10 & 2 \\
Antihipertensivo & 6 & 1 \\
Broncodilatador & 6 & 1 \\
Outros & 7 & 2 \\
\hline
\end{tabular}

dores principalmente, $\mathrm{e}$ as doenças que acometem as lactantes podem interferir negativamente na prática do aleitamento materno, pois causam estresse e desconforto, tornando necessário o tratamento medicamentoso, seja sintomático ou específico para a resolução dos problemas de saúde da lactante.

Portanto, todos os cuidados devem ser tomados quanto à saúde do binômio mãe-filho durante o período de lactação. Especificamente quanto ao tema deste estudo, o diagnóstico das doenças maternas intercorrentes durante a amamentação, o conhecimento de publicações sobre os novos medicamentos que rapidamente surgem no mercado e a utilização de medicamentos compatíveis com o aleitamento materno são fundamentais para a tomada de decisões. Deve ser destacada também a importância da divulgação de conhecimentos sobre a fisiologia da absorção e da excreção dos medicamentos, de suas propriedades farmacológicas e características bioquímicas e de todos os fatores relativos ao leite, à mama, à mãe a ao lactente, que estão envolvidos no uso de medicamentos pela lactante.

Destaque deve ser dado à orientação para que todo e qualquer tratamento da nutriz seja feito sob a supervisão e responsabilidade de um profissional médico, desestimulando o perigoso hábito da automedicação, que, no presente estudo, foi referido por apenas 25 (7\%) lactantes. Tais medidas são de extrema relevância e devem ser adotadas em todos os serviços de atendimento à saúde da gestante e da lactante, no sentido de minimizar os efeitos negativos tanto da utilização do medicamento, quanto da possibilidade de desmame em situações duvidosas e até mesmo injustificadas ${ }^{(13,14)}$.
8. Berridge $\mathrm{K}, \mathrm{McF}$ adden $\mathrm{K}$, Abayomi J, Topping J. Views of breastfeeding difficulties among drop-in-clinic attendees. Matern Child Nutr 2005;1:250-62.

9. Del Ciampo LA, Ricco RG, Almeida CAN. Aleitamento materno: passagens e transferências. São Paulo: Atheneu; 2004.

10. Schirm E, Schwagermann MP, Tobi H, de Jong-van den Berg LT. Drug use during breastfeeding. A survey from the Netherlands. Eur J Clin Nutr 2004;58:386-90.

11. McCarter-Spaulding DE. Medication in pregnancy and lactation. MCN Am J Matern Child Nurs 2005;30:10-7.

12. Pilviniene R, Maciulaitis R, Jankūnas R, Milvidaite I, Markūniene E. Breastfeeding and medications. Medicina (Kaunas) 2006;42:1035-45.

13. Lamounier JA, Cabral CM, Oliveira BC, Oliveira AB, Oliveira Jr AM, Silva AP. $O$ uso de medicamentos em puérperas interfere nas recomendações quanto ao aleitamento materno? J Pediatr (Rio J) 2002;78:57-61.

14. Chaves RG, Lamounier JA. Uso de medicamentos durante a lactação. J Pediatr (Rio J) 2004;80 (Suppl 5):S189-98. 